\title{
'Windsock' type atrial septal aneurysm in an adult causing paradoxical embolism
}

\author{
Dibbendhu Khanra ${ }^{1}$, Pradyot Tiwari ${ }^{2}$, Shishir Soni ${ }^{1}$, Anindya Mukherjee ${ }^{3}$ \\ ${ }^{1}$ Department of Cardiology, All India Institution of Medical Sciences (AIIMS), Rishikesh, India \\ ${ }^{2}$ Department of Cardiology, Apex Heart Institute, Ahmedabad, India \\ ${ }^{3}$ Department of Cardiology, Nilratan Sircar Medical College and Hospital, Kolkata, West Bengal
}

A 35-year-old female without hypertension or diabetes mellitus suffered a transient ischemic attack (TIA) with normal brain imaging. Cardiovascular examination revealed a systolic click. Transthoracic echocardiography (TTE) revealed a windsock type atrial septal aneurysm (ASA) measuring $2.3 \mathrm{~cm} \times 3.5 \mathrm{~cm}$ wallowing from the plane of inter-atrial septum (Fig. 1A) with a maximum distance of $3.5 \mathrm{~cm}$, which was popping across the tricuspid valve in diastole (Fig. 1B). Color Doppler showed bidirectional flow across a patent foramen ovale (PFO) (Fig. 1C, D). Agitated saline bubble contrast echocardiography confirmed the presence of PFO demonstrated by the passage of bubble contrasts into the left atrium within the first three cycles (Fig. 1E) and movement of ASA into the right ventricle was clearly demonstrated
(Fig. 1F; Suppl. Video 1). Three-dimensional TTE confirmed the large windsock type ASA (Fig. 1G, H) and differentiated from any intra-atrial tumor. The risk of paradoxical embolism score was 7 . Following a multi-disciplinary team discussion, a PFO closure device was offered to the patient, the patient however, did not want to undergo any procedure and she was put on apixaban $5 \mathrm{mg}$, 12-hourly. There was no recurrence of TIA in subsequent 6-month follow up. ASA is defined as a localized "saccular" deformity generally at the level of fossa ovalis. Classical 'windsock' deformity has been described in ruptured sinus of Valsalva but never in association with ASA except in one case reported in an infant. The present case of 'windsock' type ASA is the first ever case to be described in an adult presenting with paradoxical embolism according to available literature.

Conflict of interest: None declared

Address for correspondence: Dr. Dibbendhu Khanra, MD, DNB, DM Cardiology, Assistant Professor, Department of Cardiology, All India Institute of Medical Sciences, Rishikesh, Uttarakhand, India, tel: (+91)9674459039, e-mail: ddk3987@gmail.com 


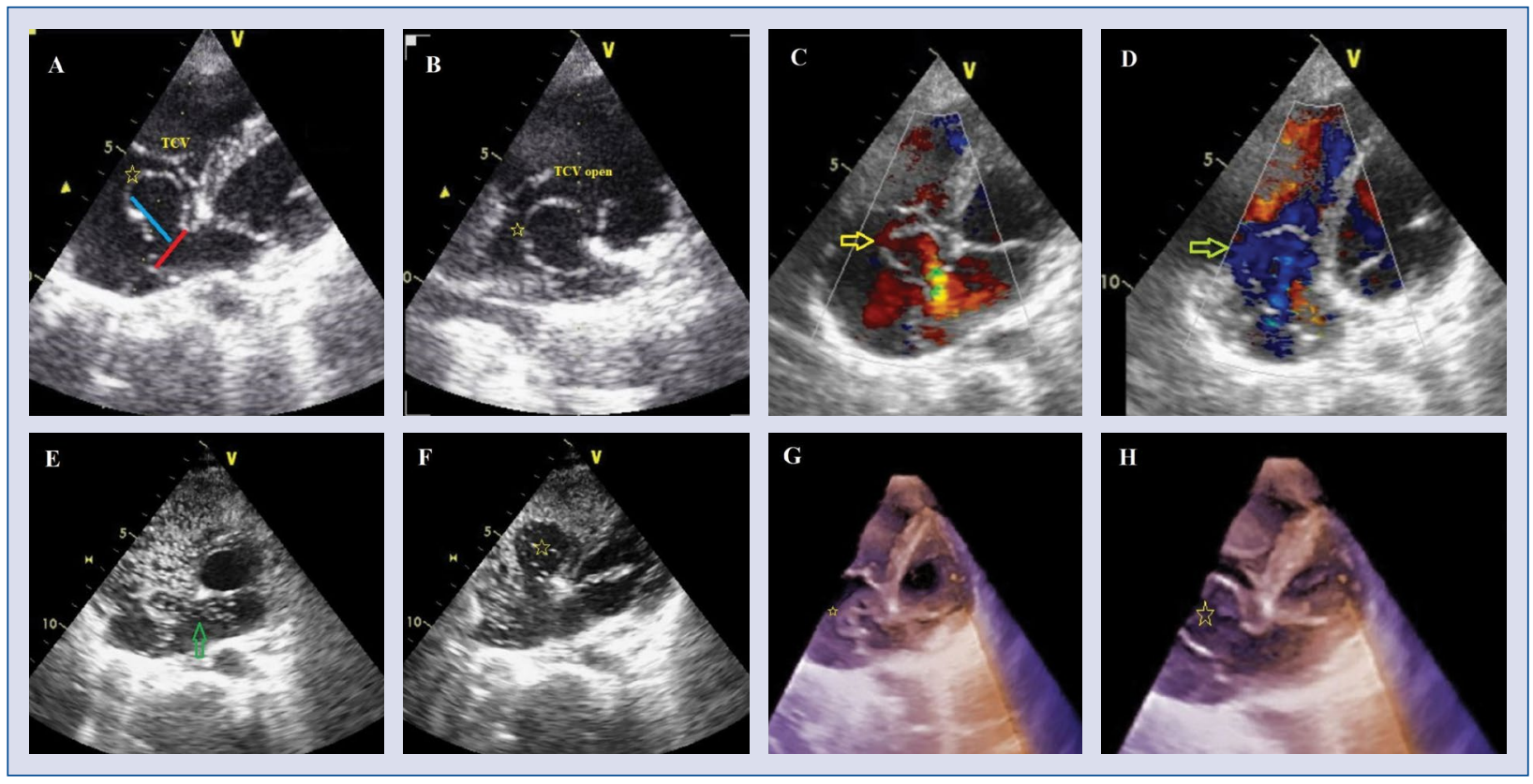

Figure 1. Transthoracic echocardiography (TTE) in modified apical four chamber view showing (A) a large 'windsock' type large atrial septal aneurysm (ASA) measuring $2.3 \mathrm{~cm} \times 3.5 \mathrm{~cm}$ (yellow asterisk), wallowing from the plane of inter-atrial septum (red line) with a maximum distance of $3.5 \mathrm{~cm}$ (blue line), which is popping across the tricuspid valve (TCV) in diastole (B); C. Color Doppler shows left to right shunt across patent foramen ovale (PFO, yellow arrow) as well as (D) right to left shunt across PFO (light green arrow); E. Agitated saline bubble contrast demonstrating PFO by the passage of bubble contrasts into the left atrium (within first three cycles) and (F) movement of ASA into the right ventricle during diastole; G, H. Three-dimensional TTE confirmed the large 'windsock' type ASA. 